\title{
Content-Based Image Retrieval Using Multiple Features
}

\author{
Chi Zhang and Lei Huang \\ School of Economics and Management, Beijing Jiaotong University, Beijing, China
}

\begin{abstract}
Algorithms of Content-Based Image Retrieval (CBIR) have been well developed along with the explosion of information. These algorithms are mainly distinguished based on feature used to describe the image content. In this paper, the algorithms that are based on color feature and texture feature for image retrieval will be presented. Color Coherence Vector based image retrieval algorithm is also attempted during the implementation process, but the best result is generated from the algorithms that weights color and texture. $80 \%$ satisfying rate is achieved.
\end{abstract}

Keywords: CBIR, color histogram, texture, color coherence vector, color feature

\section{Introduction}

With the development of Internet technology in the past 10 years, more effort is required in the process of using information due to the information explosion. The development of search engine is a nice evidence of this. Google has become the world's most innovative and dynamic IT Company because of their advanced search algorithms. However, most search engines are based on text retrieval. With the in-depth study in recent years, search algorithms have basically been able to meet the needs of people on text information retrieval. Content-based image retrieval, on the other hand, is not quite satisfying due to high technical requirements. The existing image retrieval methods are mostly done by manually entering keywords, which is trivial for untagged image search. Especially with the popularity of handheld image acquisition devices, people want to find similar images and learn about the details through their photographed image instead of typing the keyword. Content-based image retrieval (CBIR) has become critical.

\subsection{Introduction of CBIR}

Content-based image retrieval (CBIR), also known as query by image content (QBIC) [6] and content-based visual information retrieval (CBVIR), are the applications of computer vision techniques to the image retrieval problem, that is, the problem of searching for digital images in large databases. Content-based image retrieval is opposed to concept-based approaches. The main research techniques include feature extraction, similarity measurement, image matching and user feedback [4].

"Content-based" means that the search analyzes the contents of the image rather than the metadata such as keywords, tags, or descriptions associated with the image. The term "content" in this context might refer to colors, shapes, textures, or any other information that can be derived from the image itself. CBIR is desirable because searches that rely purely on metadata are dependent on annotation quality and completeness. Having humans manually annotate images by entering keywords or metadata in a large database can be time consuming and may not capture the keywords desired to describe the image. The evaluation of the effectiveness of keyword image search is subjective and has not been well-defined. In the same regard, CBIR systems have similar challenges in defining success.

The term "content-based image retrieval" seems to have originated in 1992 when it was used by T. Kato to describe experiments in automatic retrieval of images from a database, based on the colors and shapes present [1]. Since then, 
the term has been used to describe the process of retrieving desired images from a large collection on the basis of syntactical image features. The techniques, tools, and algorithms that are used originate from the fields such as statistics, pattern recognition, signal processing, and computer vision [3].

The interest in CBIR has grown because of the limitations inherent in metadata-based systems, as well as the large range of possible uses for efficient image retrieval. Textual information about images can be easily searched using existing technology, but this requires humans to manually describe each image in the database. This can be impractical for very large databases or for images that are generated automatically, e.g. those from surveillance cameras. It is also possible to miss images that use different synonyms in their descriptions. Systems based on categorizing images in semantic classes like "cat" as a subclass of "animal" can avoid the miscategorization problem, but will require more effort by a user to find images that might be "cats", but are only classified as an "animal". Many standards have been developed to categorize images, but all still face scaling and miscategorization issues [1].

Initial CBIR systems were developed to search databases based on image color, texture, and shape properties. After these systems were developed, the need for user-friendly interfaces became apparent. Therefore, efforts in the CBIR field started to include human-centered design which tried to meet the needs of the user performing the search. This typically means inclusion of query methods that may allow descriptive semantics, queries that may involve user feedback, systems that may include machine learning, and systems that may understand user satisfaction levels [3].

\subsection{CBIR Techniques}

Many CBIR systems have been developed, but the problem of retrieving images on the basis of their pixel content remains largely unsolved.

Query techniques. Different implementations of CBIR make use of different types of user queries.

Query by example is a query technique that involves providing the CBIR system with an example image that it will then base its search upon. The underlying search algorithms may vary depending on the application, but result images should all share common elements with the provided example.

Options for providing example images to the system include:

A preexisting image may be supplied by the user or chosen from a random set.

The user draws a rough approximation of the image he is looking for, for example with blobs of color or general shapes.

This query technique removes the difficulties that can arise when trying to describe images with words.

Semantic retrieval. The ideal CBIR system from a user perspective would involve what is referred to as semantic retrieval, where the user makes a request like "find pictures of Abraham Lincoln". This type of open-ended task is very difficult for computers to perform - pictures of Chihuahuas and Great Danes look very different, and Lincoln may not always be facing the camera or be in the same pose. Current CBIR systems therefore generally make use of lowerlevel features like texture, color, and shape, although some systems take advantage of very common higher-level features like faces. Not every CBIR system is generic. Some systems are designed for a specific domain, e.g. shape matching can be used for finding parts inside a CAD-CAM database.

Other query methods. Other query methods include browsing for example images, navigating customized/hierarchical categories, querying by image region (rather than the entire image), querying by multiple example images, querying by visual sketch, querying by direct specification of image features, and multimodal queries (e.g. combining touch, voice, etc.)

CBIR systems can also make use of relevance feedback, where the user progressively refines the search results by marking images in the results as "relevant", "not relevant", or "neutral" to the search query, then repeating the search with the new information.

Content comparison using image distance measures. The most common method for comparing two images in content-based image retrieval (typically an example image and an image from the database) is using an image distance measure. An image distance measure compares the 
similarity of two images in various dimensions such as color, texture, shape, and others. For example a distance of 0 signifies an exact match with the query, with respect to the dimensions that were considered. As one may intuitively gather, a value greater than 0 indicates various degrees of similarities between the images. Search results can then be sorted based on their distance to the queried image. Many measures of image distance have been developed [11].

Color. Computing distance measures based on color similarity is achieved by computing a color histogram for each image that identifies the proportion of pixels within an image holding specific values (that humans express as colors). Current research is attempting to segment color proportion by region and by spatial relationship among several color regions. Examining images based on the colors they contain is one of the most widely used techniques because it does not depend on image size or orientation. Color searches will usually involve comparing color histograms, though this is not the only technique in practice.

Texture. It measures visual patterns in images and how they are spatially defined. Textures are represented by Texel which are then placed into a number of sets, depending on how many textures are detected in the image. These sets not only define the texture, but also where in the image the texture is located.

Texture is a difficult concept to represent. The identification of specific textures in an image is achieved primarily by modeling texture as a two-dimensional gray level variation. The relative brightness of pairs of pixels is computed so that degree of contrast, regularity, coarseness and directionality may be estimated [7]. However, the problem is in identifying patterns of co-pixel variation and associating them with particular classes of textures, such as silky, or rough.

Shape. It does not refer to the shape of an image but to the shape of a particular region that is being sought out. Shapes will often be determined first by applying segmentation or edge detection to an image. Other methods like [8] use shape filters to identify given shapes of an image. In some cases accurate shape detection will require human intervention because methods like segmentation are very difficult to completely automate.

\subsection{Individual Understanding of CBIR}

Our group considers that the CBIR requires more than the match of features of the whole image. Special cases need to be treated individually.

Under some circumstances, we are more interested in specific object in the foreground or background. Meanwhile, we may also be interested in detailed information in an inconspicuous corner instead of the whole image. These cases sometimes are extremely important clues for police to solve a certain case. If we blindly pursue whole features matching, features we are really interested in may be overshadowed by other irrelevant details. This may lead to non-match in the end.

Hence interactive image retrieval is more likely to meet the needs. The interactivity requires you to not only select the most satisfying results among a plurality of results in the end, but also operations (such as the mouse click) to select the interesting area or even a symbol. It can also be used to choose the most satisfying area in the results or to speculate on the user's purpose, to adjust the relevant weight, to do more targeted search and produce more humanlike results.

Correct selection of appropriate features and the use of the mix of them is the key to featurematching.

Existing features are individually not enough to describe a whole image. If we want to achieve better search results, all aspects must be taken into account. But after various features are taken into consideration, the evaluation of the effect of the similar feature descriptions on the final result become complicated. In the experiment, we successively and separately considered color histogram, texture features and polymerization vector generated by color wavelet transform as the search features. When it comes to the mixed consideration and the regulation of weights, we realized that this will be an arduous procedure. In the actual regulation, according to existing methods, we found that this process should be an experience process and many methods require that you have a full understanding of each method first. And then you can do the weight regulation according to the large amount of experimental results. 


\section{Feature Extraction}

Feature extraction is a concept in computer vision and image processing. In computer vision and image processing, a feature is a piece of information which is relevant for solving the computational task related to a certain application. Features may be specific structures in the image such as points, edges or objects. Features may also be the result of a general neighborhood operation or feature detection applied to the image.

Other examples of features are related to motion in image sequences, to shapes defined in terms of curves or boundaries between different image regions, or to properties of such a region.

The feature concept is very general and the choice of features in a particular computer vision system may be highly dependent on the specific problem at hand.

\subsection{The Selection of the Color Space}

An important step of image processing is to choose the description method of image feature. In terms of choosing description method and feature description, it is fundamental to determine the image color space.

A color model (e.g. RGB or CMYK) is an abstract mathematical model describing the way colors can be represented as tuples of numbers, typically as three or four values or color components. However, a color model with no mapping function associated to an absolute color space is a more or less arbitrary color system with no connection to any globally understood system of color interpretation.

Adding a certain mapping function between the color model and a certain reference color space results in a definite "footprint" within the reference color space. This "footprint" is known as a gamut, and in combination with the color model, it defines a new color space. For example, Adobe RGB and sRGB are two different absolute color spaces, both based on the RGB model.

In the most generic sense of the definition above, color spaces can be defined without the use of a color model. These spaces, such as Pantone, are in effect a given set of names or numbers which are defined by the existence of a corresponding set of physical color swatches.

Color space, in accordance with the basic structure, can be divided into two categories, the primary color space and bright separate color space. The former includes RGB, the most typical one, and other types such as CMY, CMYK, $\mathrm{CIE}$ and XYZ etc. The latter includes YCC / YUV, Lab, as well as a number of hue-based color spaces. Here is a simple comparison between RGB color space and HSV space [10].

$R G B$ Color Space. The RGB color space is an additive color space in which red, green, and blue lights are added together in various ways to reproduce a broad array of colors. The name of the model comes from the initials of the three additive primary colors, red, green, and blue.

The main purpose of the RGB color space is sensing, representation, and displaying images in electronic systems, such as television and computer, though it has also been used in conventional photography. Before the electronic age, the RGB color space already had a solid theory behind it, based on human perception of colors.

RGB is a device-dependent color space: different devices detect or reproduce a given RGB value differently, since the color elements (such as phosphors or dyes) and their response to the individual $\mathrm{R}, \mathrm{G}$, and $\mathrm{B}$ levels vary from manufacturer to manufacturer, or even in the same device over time. Thus an RGB value does not define the same color across devices without some kind of color management.

Typical RGB input devices are color TVs, video cameras, image scanners, and digital cameras. Typical RGB output devices are TV sets of various technologies (CRT, LCD, plasma, etc.), computer and mobile phone displays, video projectors, multicolor LED displays, and large screens such as Jumbo Tron. Color printers, on the other hand, are not RGB devices but subtractive color devices (typically CMYK color space).

This paper discusses concepts common to all the different color spaces that use the RGB color space, which are used in one implementation or another in color image-producing technology.

HSV Color Space. HSV is the most common cylindrical-coordinate representation of points in an RGB color model. Developed in the 1970s for computer graphics applications, HSV is used 
today in color pickers, in image editing software, and less commonly in image analysis and computer vision.

The representation rearranges the geometry of RGB in an attempt to be more intuitive and perceptually relevant than the Cartesian (cube) representation, by mapping the values into a cylinder loosely inspired by a traditional color wheel. The angle around the central vertical axis corresponds to "hue" and the distance from the axis corresponds to "saturation". These first two values give the two schemes the ' $\mathrm{H}$ ' and ' $S$ ' in their names. The height corresponds to a third value, the system's representation of the perceived luminance in relation to the saturation.

In each cylinder, the angle around the central vertical axis corresponds to "hue", the distance from the axis corresponds to "saturation", and the distance along the axis corresponds to "lightness", "value" or "brightness". Note that while "hue" in HSV refers to the same attribute, their definitions of "saturation" differ dramatically. Because HSV are simple transformations of device-dependent RGB models, the physical colors they define depend on the colors of the red, green, and blue primaries of the device or of the particular RGB space, and on the gamma correction used to represent the amounts of those primaries. Each unique RGB device therefore has unique HSV spaces to accompany it, and numerical HSV values describe a different color for each basic RGB space.

As it can be seen by both RGB and HSV color spaces designed that RGB is the "machineoriented" color space, whose purpose is to enable the machine to accurately describe the color, while HSV should belong to the "useroriented" color space. After the test, HSV color space using the basic color histogram gave an amazing result, in the search of a single basic color, single object image. It produced more than 15 correct results in the search for a poker match on black background. The accuracy rate was also high in the search for an eagle or aircraft in the blue sky and the "error" result was the same basic color as the original image, the differences lay in the foreground objects. So here we choose HSV color space.

\subsection{Image Feature Selection}

In image processing, the concept of feature is used to denote a piece of information which is relevant for solving the computational task related to a certain application. More specifically, features can refer to the result of a general neighborhood operation (feature extractor or feature detector) applied to the image, specific structures in the image itself, ranging from simple structures such as points or edges to more complex structures such as objects.

Other examples of features are related to motion in image sequences, to shapes defined in terms of curves or boundaries between different image regions, or to properties of such a region.

A specific image feature, defined in terms of a specific structure in the image data, can often be represented in different ways. For example, an edge can be represented as a Boolean variable in each image point that describes whether an edge is present at that point. Alternatively, we can use a representation which provides a certain measure instead of a Boolean statement of the edge's existence and combine this with information about the orientation of the edge. Similarly, the color of a specific region can either be represented in terms of the average color or a color histogram.

Color feature, texture feature, shape feature and characteristics of spatial relationship are commonly used image features [9].

Color Feature. Color feature is the most intuitive and obvious feature of the image, and generally adopts histograms to describe it. Commonly used methods are based on color features: color histogram matching method, the color sets, color moments, color coherence vector, color related maps. Color histograms method has the advantages of speed, low demand of memory space and insensitivity to the images' changes of the size and rotation, it consequently wins extensive attention.

The strategy adopted in color feature selection is the use of 36-dimensional color feature vector. It is described as three basic parameters $\mathrm{H}, \mathrm{S}, \mathrm{V}$ and combinations of two HS, HV, SV to count the number of pixels, which are located in the interval $(0,43),[43,85),[85,128),[128,170)$, $[170,213),[213,255)$. Then the distance between two vectors is calculated, the closer vectors have higher similarity. Ultimately, the top 20 are selected as a result. 
Texture Feature. When it refers to the description of the image's texture, we usually adopt texture's statistic feature and structure feature as well as the features based on special domain that are changed into frequency domain. This method uses Haar wavelet transform to extract the image texture features, which uses wavelet basis instead of Fourier basis to observe a whole signal space by scaling or shifting. The disadvantage of the Fourier transform is its inability to reflect the local characteristics of the signal. Using Matlab's own function, wavelet statistical features in HSV space are calculated as basic texture features. Then we choose the appropriate distance metric (for metric see details) to obtain a more similar image as a result.

Besides the above-mentioned disadvantages of texture features, we still choose texture features as the image feature for the following reasons:

1. In this experiment, the problems listed above were not a problem, because the images in the search image database were specially selected, with resolution $384 * 256$ or $256 * 384$. Effects of the same resolution on the actual results can be negligible.

2. The texture changes are affected by the light generated. The $\mathrm{V}$ property in HSV color space is the color of the bright. This "bright" does not mean the light intensity. There is no way to overcome this shortcoming temporarily. But later tests showed interesting results. After a preliminary color characteristics screening, the use of the texture features is better than the use of color feature or texture feature alone. Texture is an effective approach when the search images have great differences in thickness, density, etc. Texture features are usually very difficult to accurately reflect human visual perception between different textures. When the thickness, density and other texture information which are easy to distinguish are very close, the actual tests found that when all the images are part of an object (such as cell photomicrograph), adding texture information search results is significantly better than the color information alone. It also found that, in some cases, search results after adding texture information are worse than by using only color feature approach. These cases mainly occur in the search that has obvious difference between foreground and background. The background texture information will lead to the search results to have the same background and different prospects. This problem is more serious than only occasionally using the color feature. In search of some oil painting images, which have a strong sense of reality, the texture information will mislead the method to search the oil painting content instead to only search paintings.

Of course, different evaluation criteria of the search have different results. When you want to search for the oil content, the result is better. While for the other paintings, the same results will become poor. This situation exists in various methods.

Color Coherence Vector. As the depth application of the color features, color coherence vector (CCV) [5] not only takes into account the number of pixel points in different colors, but also the relative position information of the pixels [10]. Most of the early color features reflect the color information, but not the color spatial distribution. The effect is different for different distribution of pixels of the same color and quantity. Based on this consideration, the image feature information of the CBIR polymerizes with color vector as an alternative feature [4].

Color coherence vector is an improved conventional histogram. The pixel points belonging to color bucket of the same color histogram are divided into coherence and incoherence, which refers to pixels of the same color distributed over the same area.

We can obtain color coherence vector through statistics of pixels of more or less the same color than the threshold value in each color. Algorithm uses eight colors of red, green, blue, yellow, purple, blue, white and black.

\section{Similarity Measure}

This method is to rank previous search results by calculating the eigenvector distance of two images.

\subsection{Taxicab Geometry}

Taxicab geometry, created by Hermann Asstitski in the 19th century Germany [2], is a form of geometry in which the usual distance function 
or metric of Euclidean geometry is replaced by a new metric in which the distance between two points is the sum of the absolute differences of their Cartesian coordinates. The taxicab metric is also known as rectilinear distance, $\mathrm{L} 1$ distance or $\ell_{1}$ norm, city block distance, Manhattan distance, or Manhattan length, with corresponding variations in the name of the geometry. The latter names allude to the grid layout of most streets on the island of Manhattan, which causes the shortest path a car could take between two intersections in the borough to have length equal to the intersections' distance in taxicab geometry.

The taxicab distance, $d_{1}$, between two vectors $\mathbf{p}, \mathbf{q}$ in an $n$-dimensional real vector space with fixed Cartesian coordinate system, is the sum of the lengths of the projections of the line segment between the points onto the coordinate axes. More formally,

$$
d_{1}(\mathbf{p}, \mathbf{q})=\|\mathbf{p}-\mathbf{q}\|_{1}=\sum_{i=1}^{n}\left|p_{i}-q_{i}\right|
$$

$\mathbf{p}=\left(p_{1}, p_{2}, \ldots, p_{n}\right)$ and $\mathbf{q}=\left(q_{1}, q_{2}, \ldots, q_{n}\right)$. For example, in the plane, the taxicab distance between $\left(p_{1}, p_{2}\right)$ and $\left(q_{1}, q_{2}\right)$ is $\left|p_{1}-q_{1}\right|+\mid p_{2}-$ $q_{2} \mid$.

The following chart shows search results in three methods with Manhattan distance: test 1 is a painting of trees and peaks; test 21 is a puppet on the white background; test 33 is a microscopic structure of a cell; test 38 is poker king; test64 is several flower mushrooms in the forest; and test 212 is a beauty.

\begin{tabular}{|l||c|c|c|}
\hline Test & Color & Color + Texture & CCV \\
\hline \hline Test1 (painting) & 5 & 5 & 1 \\
\hline Test21 (puppet) & 14 & 14 & 8 \\
\hline Test33 (cell) & 2 & 2 & 0 \\
\hline Test38 (poker king) & 11 & 11 & 3 \\
\hline Test64 (mushrooms) & 6 & 6 & 1 \\
\hline Test212 (beauty) & 2 & 4 & 2 \\
\hline
\end{tabular}

Table 1. Search results in three methods with Manhattan distance.

\subsection{Euclidean Distance}

In mathematics, the Euclidean distance or Euclidean metric is the "ordinary" distance between two points that one would measure with a ruler, and is given by the Pythagorean formula [2].

The Euclidean distance between points $\mathbf{p}$ and $\mathbf{q}$ is the length of the line segment connecting them $(\overline{\mathbf{p q}})$.

In Cartesian coordinates, if $\mathbf{p}=\left(p_{1}, p_{2}, \ldots, p_{n}\right)$ and $\mathbf{q}=\left(q_{1}, q_{2}, \ldots, q_{n}\right)$ are two points in Euclidean $n$-space, the distance from $\mathbf{p}$ to $\mathbf{q}$, or from $\mathbf{q}$ to $\mathbf{p}$ is given by:

$$
\begin{aligned}
& d(\mathbf{p}, \mathbf{q})=d(\mathbf{q}, \mathbf{p}) \\
& =\sqrt{\left(q_{1}-p_{1}\right)^{2}+\left(q_{2}-p_{2}\right)^{2}+\ldots+\left(q_{n}-p_{n}\right)^{2}} \\
& =\sqrt{\sum_{i=1}^{n}\left(q_{i}-p_{i}\right)^{2}}
\end{aligned}
$$

The position of a point in a Euclidean $n$-space is a Euclidean vector. So, $\mathbf{p}$ and $\mathbf{q}$ are $\mathrm{Eu}-$ clidean vectors, starting from the origin of the space, and their tips indicate two points. The Euclidean norm, or Euclidean length, or magnitude of a vector measures the length of the vector:

$$
\|\mathbf{p}\|=\sqrt{p_{1}^{2}+p_{2}^{2}+\ldots+p_{n}^{2}}=\sqrt{\mathbf{p} \cdot \mathbf{p}}
$$

The following chart shows search results in three methods with Euclidean distance.

\begin{tabular}{|l||c|c|c|}
\hline Test & Color & Color + Texture & CCV \\
\hline \hline Test1 (painting) & 4 & 4 & 1 \\
\hline Test21 (puppet) & 11 & 11 & 7 \\
\hline Test33 (cell) & 6 & 6 & 0 \\
\hline Test38 (poker king) & 10 & 9 & 3 \\
\hline Test64 (mushrooms) & 2 & 2 & 4 \\
\hline Test212 (beauty) & 3 & 3 & 0 \\
\hline
\end{tabular}

Table 2. Search results in three methods with Euclidean distance.

\section{CBIR Using Multi Features}

Euclidean distance and the Manhattan distance formula reveal that the Manhattan distance weakens the influence of the element size of main 
components by removing the square of Euclidean distance, while Manhattan distance considers more colors in search results, by withdrawing the reward towards less differentiated colors. In comparison to Euclidean distance, the Manhattan distance is preferable.

Using Manhattan distance as a measure, color features, texture features and color coherence vector as criteria (Color feature has an exaggeration of 20 times, texture feature 4 magnification, distance of color coherence vector remains constant), the results are as follows:

\begin{tabular}{|c||c|}
\hline Feature & Color + Texture + CCV \\
\hline \hline Test1 (painting) & 5 \\
\hline Test21 (puppet) & 14 \\
\hline Test33 (cell) & 2 \\
\hline Test38 (poker king) & 11 \\
\hline Test64 (mushrooms) & 5 \\
\hline Test212 (beauty) & 2 \\
\hline
\end{tabular}

Table 3. Search results in the integration of three methods in Manhattan distance.

In addition, due to the inconsistency in color and texture features metric, the calculation of the Euclidean distance will cause some additional effects.

Therefore, Manhattan distance is chosen to measure the distance. It is recommended to apply the measurement of methods' similarity based on calculating the cosine value. More formally,

$$
\begin{gathered}
\operatorname{Sim}\left(X, X^{\prime}\right)=\frac{X \cdot X^{\prime}}{|X| \cdot\left|X^{\prime}\right|} \\
X=\left(x_{1}, x_{2}, \ldots, x_{m}\right), X^{\prime}=\left(x_{1}^{\prime}, x_{2}^{\prime}, \ldots, x_{m}^{\prime}\right) \\
X \cdot X^{\prime}=\sum_{i=1}^{m} x_{i} x_{i}^{\prime}
\end{gathered}
$$

Several tests show the following results:

Remark 1. Color feature outperformed the other features in relatively simple background and clear foreground objects, but texture features perform well when the photograph is part of an object.

Remark 2. Based on the selected criteria, color and texture fused method performs better than others. Some search results of fused method and single method have the same feature number, but the ranked result of fused method has better feature under our requirements (When searching for poker King, high on the list are poker Jack, Queen, King). Experimental results reveal that adding CCV does not improve the accuracy. It also shows that CCV computational experiments are based on the RGB color space at first, which failed to produce a satisfactory answer. Extraction process is timeconsuming and the effect is not obvious, therefore, $\mathrm{CCV}$ feature is removed (it took 3 days to extract 9500 images, each image only extracted 16-dimensional vector).

\section{Image Matching}

Feature extraction of selected images has been done previously and results are saved to .mat files.

Two options were considered during image matching:

1. Add appropriate weight values for distance calculation. Then matching results are determined by distance.

2. Obtain several former images as search results using distance calculation with color feature and texture feature.

In "weights strategy" matching test, color feature vector distance is magnified 10 times, texture feature vector distance remains unchanged. Color feature vector distance is magnified 20 times, texture feature vector distance is magnified 5 times. Color feature vector distance is magnified 20 times, texture feature vector distance remains unchanged. The second method achieved the best results in the test.

In "priority color" matching test, 900, 500, 300, 100 and 50 are tested to select "n". Searching

\begin{tabular}{|c||c|c|}
\hline Strategy & $\begin{array}{c}\text { "Weights } \\
\text { strategy" }\end{array}$ & $\begin{array}{c}\text { "Priority } \\
\text { color" }\end{array}$ \\
\hline \hline Test1 (painting) & 5 & 4 \\
\hline Test21 (puppet) & 16 & 16 \\
\hline Test33 (cell) & 2 & 4 \\
\hline Test38 (poker king) & 19 & 15 \\
\hline Test64 (mushrooms) & 5 & 4 \\
\hline Test212 (beauty) & 4 & 2 \\
\hline
\end{tabular}

Table 4. Search results with a different matching. 
results become better as " $n$ " decreases, which shows that color feature plays a more important role in selecting color and texture feature.

Table 4 contains the results of different matching strategy.

\section{Conclusions}

Most of the experimental results are explained in the method selection process, the following section is brief review.

Figures 1 and 2 below show the screenshot of search results. Figure 1 is the result of the poker

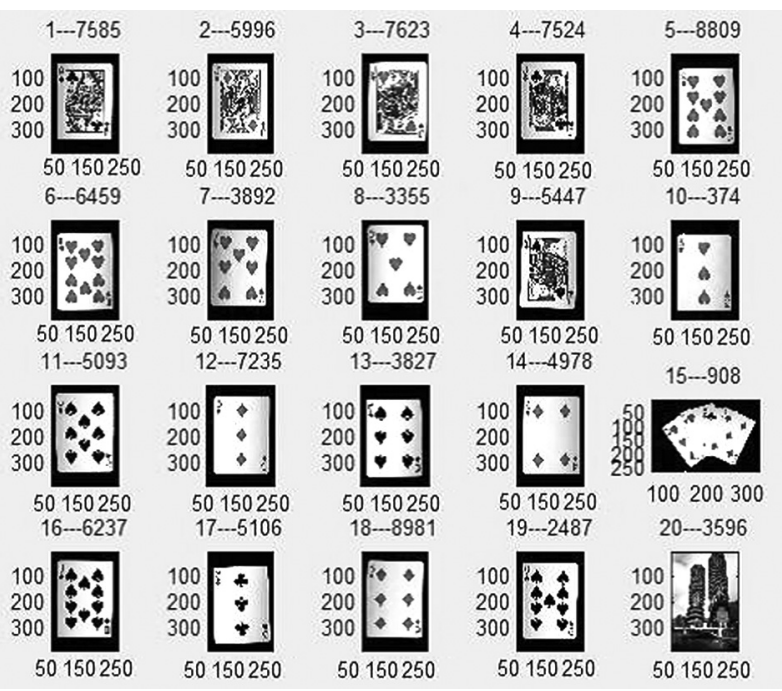

Figure 1. The search results of Test 38 .

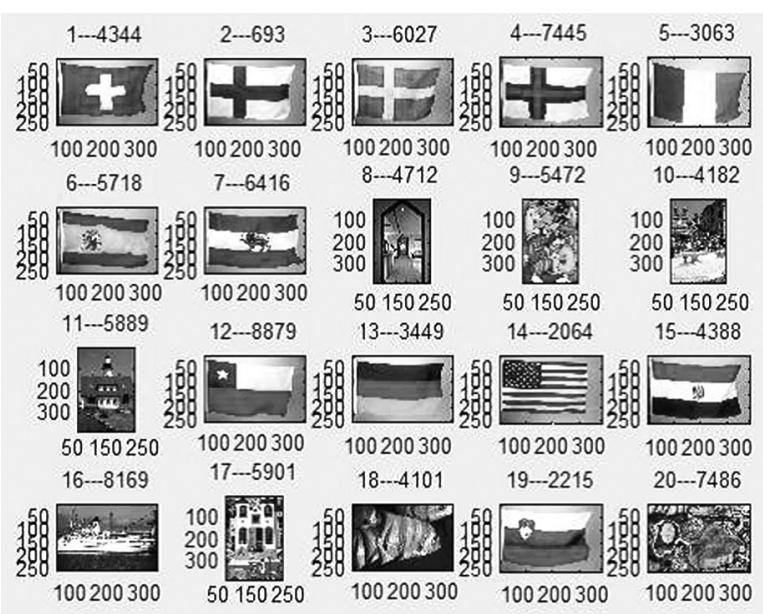

King, and figure 2 is the result of the Six-color flag. Both of them have simple background.

Satisfactory results are achieved when searching image is with simple background. But background contributes more to the result when searching object is in complex background. According to Figure 3, searching results mostly have different foreground and similar background.

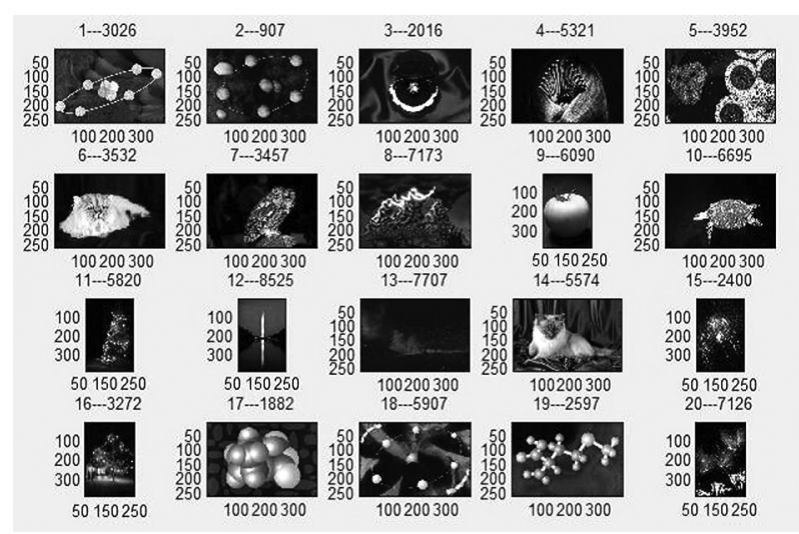

Figure 3. The search results of Test55.

The method used in the experiment is relatively simple, and the model is conservative.

In this paper, we proposed a multi feature model for the Content-Based Image Retrieval System by combining the color, texture, and Color Coherence Vector. Users were given options to select the appropriate feature extraction method for best results. The results are quite good for most of the query images which have simple background and it is possible to improve them further by tuning the threshold and adding relevance feedback. In this dissertation the Manhattan distance is calculated between the two pixels of the matching images.

\section{Acknowledgments}

This research was supported by "Research of Logistics Resource Integration and Scheduling Optimization" under the National Natural Science Foundation 71132008. Thank you.

Figure 2. The search results of Test 211. 


\section{References}

[1] J. EAKINS, M. GRAHAM, Content-based Image Retrieval. University of Northumbria at Newcastle, 2014.

[2] E. F. Krause, Taxicab Geometry: Adventure in Non-Euclidean Geometry. Dover Publications Inc, January, 1987.

[3] M. S. Lew, N. Sebe, C. DJeraba, Content-based multimedia information retrieval: State of the art and challenges. Iit ACM Transactions on Multimedia Computing, Communications, and Applications (TOMCCAP), 2(1) (2006), 1-19.

[4] F.-J. MENG, B.-L. GuO, Research on Content-Based Image Retrieval Technology. Application Research of Computers, 21(7) (2004).

[5] G. PASS, R. ZABIH, J. Miller, Comparing images using color coherence vectors. Proceedings of the fourth ACM International Conference on Multimedia, ACM, (1997) pp. 65-73.

[6] Y. RuI, T. S. HuANG, S. F. CHANG, Image retrieval: Current techniques, promising directions, and open issues. Journal of Visual Communication and Image Representation, 10(1) (1999), 39-62.

[7] H. TAMURA, S. Mori, T. YAMAWAKI, Textural features corresponding to visual perception. Systems, Man and Cybernetics, IEEE Transactions on, 8(6) (1978), 460-473.

[8] F. Tushabe, M. H. F. Wilkinson, Content-based image retrieval using combined $2 D$ attribute pattern spectra, Advances in Multilingual and Multimodal Information Retrieval. Springer Berlin Heidelberg, 2008.

[9] X. Wang, F. Hu, H. Yang, Region-Based Color Image Retrieval Using Color and Texture Features. Journal of Chinese Computer Systems, 28(2) (2007).

[10] Y. Zhang, Y. XU, H. BaO, Offline Handwritten Signature Recognition Method Based on Multifeatures. JCIT, 8(5) (2013), 538-546.

[11] H. EIDENBERGER, Fundamental Media Understanding. Atpress, (2011).
Received: October, 2013 Revised: April, 2014 Accepted: July, 2014

Contact addresses:

Chi Zhang

School of Economics and Management Beijing Jiaotong University

Beijing, 100044

China

e-mail: crazyzhc98@gmail.com

Lei Huang

School of Economics and Management Beijing Jiaotong University Beijing, 100044

e-mail: lhuang@bjtu.com

CHI ZHANG is a PHD candidate at the School of Economics and Management of Beijing Jiaotong University. He was born in Zhengzhou, Henan, in 1990. He graduated from the Department of Automation Engineering of the School of Electronic and Information Engineering at Beijing Jiaotong University in 2008. Since 2012, his main research interest has been Information management. His PhD mentor is Professor Huang.

LEI HUANG is a professor and $\mathrm{PhD}$ mentor at the School of Economics and Management of Beijing Jiaotong University. He was born in 1965. In 1989, he joined Beijing Jiaotong University and has been working there until now. His research interests lie on Management theory and methods and Information technology planning and design. 